\title{
The formation of rhythmic categories and metric priming ${ }^{\dagger}$
}

\author{
Peter Desain, Henkjan Honing $₫$ \\ Music, Mind, Machine Group, NICl, University of Nijmegen, PO Box 9104, 6500 HE Nijmegen, \\ The Netherlands; - also at Music Department/ILLC, University of Amsterdam, Spuistraat 134, \\ 1012 VB Amsterdam, The Netherlands; e-mail: desain@nici.kun.nl; honing@hum.uva.nl \\ Received 8 April 2002, in revised form 4 November 2002; published online 20 February 2003
}

\begin{abstract}
Two experiments on categorical rhythm perception are reported, the object of which was to investigate how listeners perceive discrete rhythmic categories while listening to rhythms performed on a continuous time scale. This is studied by considering the space of all temporal patterns (all possible rhythms made up of three intervals) and how they, in perception, are partitioned into categories, ie where the boundaries of these categories are located. This process of categorisation is formalised as the mapping from the continuous space of a series of time intervals to a discrete, symbolic domain of integer-ratio sequences. The methodological framework uses concepts from mathematics and psychology (eg convexity and entropy) that allow precise characterisations of the empirical results.

In the first experiment, twenty-nine participants performed an identification task with 66 rhythmic stimuli (a systematic sampling of the performance space). The results show that listeners do not just perceive the time intervals between onsets of sounds as placed in a homogeneous continuum. Instead, they can reliably identify rhythmic categories, as a chronotopic time clumping map reveals. In a second experiment, the effect of metric priming was studied by presenting the same stimuli but preceded with a duple or triple metre subdivision. It is shown that presenting patterns in the context of a metre has a large effect on rhythmic categorisation: the presence of a specific musical metre primes the perception of specific rhythmic patterns.
\end{abstract}

\section{Introduction}

Time, as a subjective structuring of events in music, is quite different from the concept of time as duration in the physical realm (Michon and Jackson 1985; Jones 1990; Large and Jones 1999). Listeners to music do not perceive the time intervals between onsets of sounds as being placed in a homogeneous continuum. Instead, they identify rhythmic pattern, categories that function as a reference relative to which deviations from strict mechanical timing can be appreciated (Desain and Honing 1992; Clarke 1999; Desain and Windsor 2000). In fact, temporal patterns in music combine two representations of time that are essentially different: the discrete rhythmic durations as symbolised by, for example, the notes in a musical score, and the continuous timing variations that characterise an expressive musical performance. In music performance, timing information is added to the nominal durations of the note categories, based on the interpretation of the musician (see figure 1a). These deviations are experienced as the expressive character of the performed rhythm (eg 'anticipated' or 'swinging') and they are to a large extent relative to the structure of the music, such as musical phrases, metre, or rhythmic structure. These structural and expressive properties of musical rhythm have been studied extensively (Palmer 1997; Clarke 1999; Gabrielsson 1999). In the perception of music (see figure 1b), the listener separates this temporal information into rhythmic categories and expressive timing. For example, listeners will recognise the performed rhythm shown in figure $1 \mathrm{a}$ as the one notated in figure $1 \mathrm{~b} .{ }^{(1)}$ Next to the

$\dagger$ Both authors contributed equally to this work.

(1) Note that, in common music notation (CMN), several notational variances of the same underlying integer representation are possible: the notation shown is an often encountered one. 


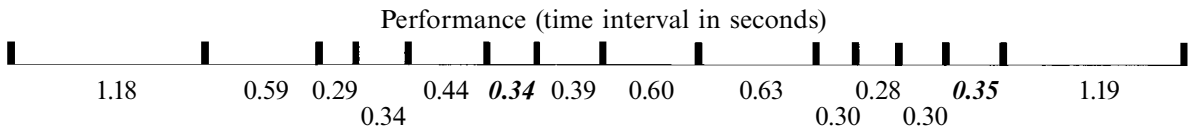

(a)
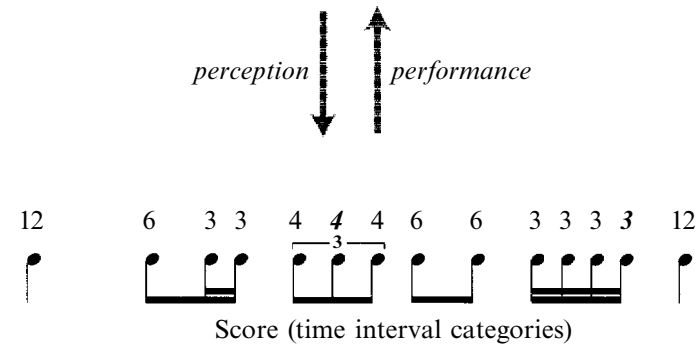

(b)

Figure 1. Example of the two representations of time present in music: a performed rhythm in continuous time (a) and the perceived rhythmic interpretation in discrete, symbolic time (b). (An audio example is available at www.nici.kun.nl/mmm/time.html.)

recognition of these discrete rhythmic durations, a listener still perceives the expressive timing of the performed rhythm. Even untrained listeners appreciate the 'against the beat' quality of the triplets (Vos and Handel 1987) and the natural slowing down at the end of the group of sixteenth notes (Todd 1992). And one can argue that, to be able to perceive a long note as a local slowing down of tempo, one needs a reference, which by itself suggests the existence of rhythmic categories.

The first question to be addressed in this paper concerns the mapping of the continuous space of performed temporal patterns to the discrete, symbolic space of rhythmic categories (the mapping from figure 1a to figure 1b). This issue has been treated in a relatively small number of studies on categorical rhythm perception (Clarke 1987; Schulze 1989). For a few special cases, a sharp transitional boundary between rhythmic categories has been found, with a high sensitivity for differences around this boundary. These studies will be reviewed in more detail in section 2.5. The second question to consider deals with the factors that influence this mapping, in particular the metrical context in which the performed patterns are presented. With regard to this influence of metre, Clarke (1987) has presented some evidence showing that the position of the boundary between rhythmic categories may shift depending on the metrical context in which the rhythm is presented. In studies by Clarke and by Schultze, however, only few categories were investigated. In contrast, in this study, we systematically consider a large set of temporal patterns and the effect of metre on their identification.

In this paper, which is a result of interdisciplinary research, we use a number of concepts and analysis tools that are relatively new to rhythm-perception research. Some of them have already been of use in other fields of perception, for instance the use of maps in the study of colour vision (Le Grand 1968). Others stem from physics (eg the concept of entropy) or mathematics (eg the notion of abstract space and of convexity). In introducing their use, we hope to demonstrate how they help to arrive at the abstract levels of understanding needed to build general theories of human rhythm perception, which is a beautiful, and beautifully complex, process.

\section{Rhythmic categories and expressive timing}

\subsection{Categorisation}

We use the term categorisation to describe the cognitive process of extracting the discrete rhythmic categories from the continuous signal. The continuous time intervals in music performance are not just categorised into symbolic categories: the categories themselves have structure, as they relate to each other as rational numbers. They can 
be represented and coded by small integers, each signifying a multiple of a small symbolic duration. Thus, because of the suspected context dependence, we will consider categorisation of temporal sequences as a whole, not of individual performed time intervals. We expect the series of categories that result from this cognitive process to exhibit structural regularities.

In the rhythm perception literature, there are different approaches to this phenomenon. Fraisse (1982), for instance, stressed the importance of low integer ratios (like 1: 1 and $1: 2$ ) in the perception of rhythm, ratios to which non-integer rhythms will migrate. Other authors (eg Nakajima 1987) suggest that categorisation is in fact a mapping from a single continuous time dimension into categorical intervals, independent of context. Still others (eg Clarke 1987) investigated whether this categorisation might in fact be a result of categorical perception (we return to this topic in section 2.2). However, the implication of true categorical perception is that expressive timing would be barely detectable, which clearly is not the case (Clarke 1999). In contrast, it has been argued (eg Clarke 2000) that categorisation is not simply a mapping from a continuous variable to a discrete one (losing important continuous information in the process), but that both types of information (ie the rhythmic category and expressive timing) are available at the same time. But we will argue that expressive timing is only perceivable because there is categorisation, the categorisation functioning as a reference relative to which timing deviations are perceived. In this view, both types of information are available to the listener, with categorisation determining the expressive timing perceived.

In the categorisation of a time interval, it has been shown that neighbouring intervals play an important role (Desain and Honing 1991). As an example, consider the two marked (bold italic) time intervals in figure 1. While the first note is played slightly shorter than the second, the former is commonly interpreted as longer by a third (ie the first as part of a triplet, the second as a sixteenth note). This typical example illustrates that categorisation is a cognitive process that cannot be described by some simple round-off procedure: the local temporal context has to be taken into account to explain the perceived duration of the time interval. Furthermore, not only is there dependence on context in rhythm perception: without context, categorisation is hardly possible. Sternberg et al (1982) showed that even experienced musicians have difficulty in recognising and reproducing relatively simple ratios like $1: 5$ or $3: 4$ if they are presented in isolation. While in music practice these ratios (and even much more complex ones) are quite common, one always needs some context to be able to recognise and perform them well.

\subsection{Categorical rhythm perception}

Categorical rhythm perception (Clarke 1999) has been studied by presenting interpolations between different rhythmic patterns to listeners in both an identification task (probing the recognition of categories) and a discrimination task (testing for increased sensitivity near category boundaries). As such, it applies the paradigm developed for categorical perception (Harnad 1987) as used, for instance, in the domains of speech (Repp 1984) and colour perception (Saunders and van Brakel 1997). While categorical perception experiments in these domains have sometimes been used to determine whether this process is innate or learned, it was shown by Clarke (1987) that, at least for the rhythms studied, it is open to top-down cognitive influence. Clarke (1987) describes an experiment in which a short musical sequence was presented in two different metrical contexts $(2 / 8$ and $3 / 8$ metre, ie a duple and a triple metre), with the two notes at the end of the sequence systematically varied between the ratios $1: 1$ and $1: 2$ (see figure 2a). ${ }^{(2)}$ The participants performed an identification task in which they had to identify the rhythm as belonging to type $1: 1$ or type $1: 2$, and a discrimination

(2) Note that note symbols are used throughout to represent inter-onset intervals; hence the last note is notated as durationless (ie marked with a cross). 


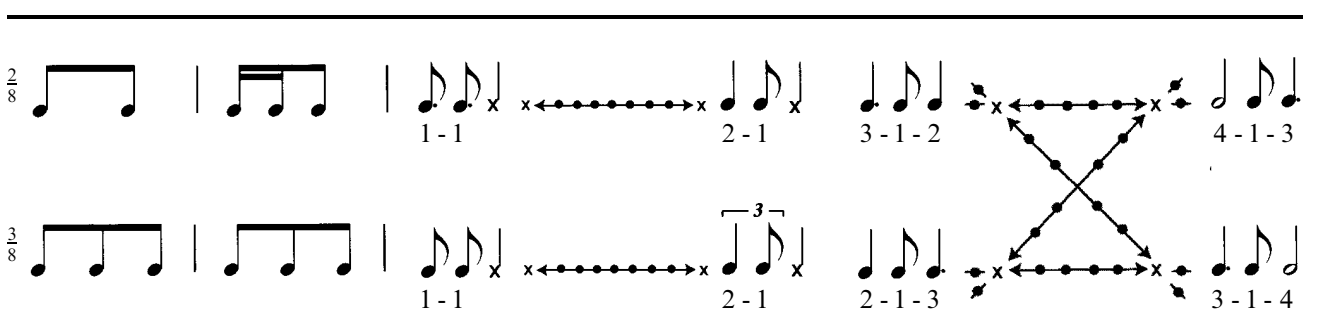

(a)

(b)

Figure 2. Stimuli used by (a) Clarke (1987) and (b) Schulze (1989) (see text for details).

task in which they judged whether a pair of rhythms was same or different. The resulting identification function showed a strong change in slope at the category boundary between the two rhythms, and the discrimination function has a strong peak in the same position, which, as such, is clear evidence for categorical perception. Shultze (1989) did a follow-up study addressing some of the methodological problems of Clarke's study, the main point being that the forced-choice paradigm used in the identification task steered the participant's responses towards the available categories. Schulze therefore used a somewhat different experimental setup, in which he trained two participants with a set of interpolated rhythms, and asked them to give a graded identification response (ie as many response categories as stimulus types). The discrimination function was derived indirectly from these responses. He investigated interpolations between four different 'prototypical' rhythms (see figure 2b). The patterns were not preceded by a metrical context, as in Clarke (1987). However, he found similar effects, although weaker for some rhythmic patterns. Furthermore, he showed that categorical rhythm perception is open to perceptual learning (cf Livingstone et al 1998), the participants being able to distinguish more categories after an intensive training period prior to the experimental trials.

These studies provide evidence for the categorical perception of rhythmic patterns. Their local character leaves open the question to what extent the results can be generalised for the large variety of patterns occurring in real music, and how the shape and position of these categories are affected by metre (a boundary shift was only shown for the rhythmic categories 1-1 and 2-1). We will therefore conduct a systematic categorisation study and use a large set of temporal patterns as stimuli, patterns of four note onsets and a fixed total duration of $1 \mathrm{~s}$ (sampled on a fine temporal grid). These will be presented without metrical context (experiment 1) and in three metrical contexts (no metre, duple metre, and triple metre; experiment 2), as the participant engages in an identification task with semi-open responses.

To study the process of categorisation as described above, we need a formal and computational framework. For this, we will introduce the abstract notion of rhythm spaces and conceptualise categorisation as a mapping operation between them. Consequently, these notions will be used in the analyses of the experimental results.

\subsection{Representing temporal patterns: The performance space}

Instead of studying an arbitrarily chosen set of rhythmic patterns, we consider the space of all possible performances of $n$ time intervals. In this $n$-dimensional space, every point represents a different temporal pattern. This infinite set contains both musical and unmusical rhythms, and captures, in principle, all possible performances of all rhythms of $n+1$ onsets. We will therefore refer to this set as the performance space. Restricting ourselves to four onsets, we can represent any pattern in a three-dimensional space (see figure 3a), with the three axes representing the three inter-onset intervals (IOIs). All patterns that add up to a fixed total duration form a diagonal triangular slice in such a space. If one looks from above, towards the origin, the triangle can be presented as a ternary plot (see figure $3 b$ ). 


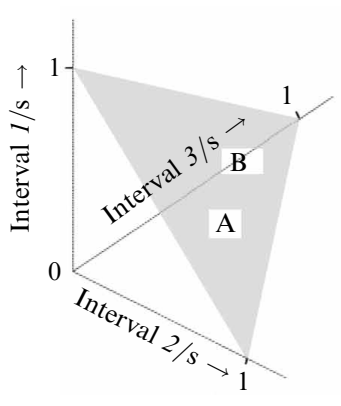

(a)

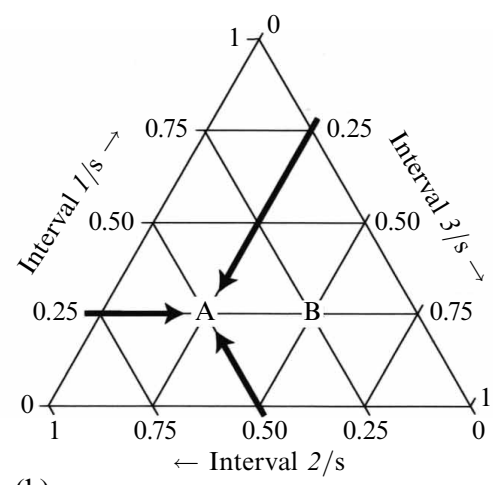

(b)

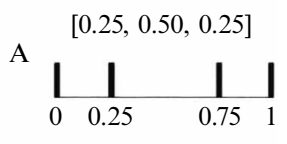

B

$[0.25,0.25,0.50]$

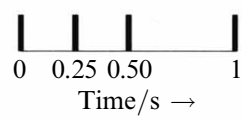

(c)

Figure 3. Performance space of all three-interval temporal patterns adding up to $1 \mathrm{~s}$ duration (a), ternary plot (b), and two example patterns (c) (see text for details).

This ternary plot, which depicts dependent three-dimensional data, still allows the determination of the coordinates of the points: a specific performance can be located by reading the grid along the direction of the tick marks at the axes, with interval size (or note duration) depicted clockwise and interval number depicted counterclockwise. For example, the point labeled A corresponds to the pattern (shown in figure 3c, its first IOI being 0.25 , the second being 0.5 , and the last IOI $0.25 \mathrm{~s}^{(3)}$ We will use this chronotopic map to represent our stimuli and the way they are perceived.

As an example, and for comparison with the empirical data discussed in section 2.2, figure 4 shows the stimuli used in Schulze (1989), depicted as a chronotopic map. The black dots identify the stimulus patterns, and interpolations between their mechanical versions are marked by crosses. The category boundaries are indicated with a gray line. The gray area is the hypothetical shape of the rhythmic category A. However, we can only base its contours on two measurements (the boundaries between $\mathrm{A}$ and $\mathrm{B}$, and $\mathrm{A}$ and $\mathrm{C}$ ), too few to infer its shape.
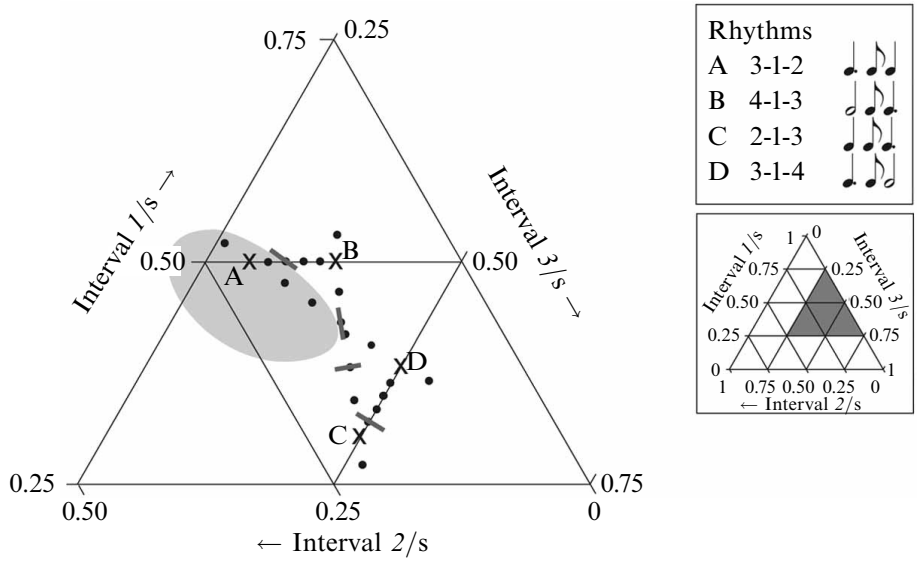

Figure 4. Stimuli and results of Schulze $(1989, N=2)$ depicted as a chronotopic map zooming-in on part of the performance space, indicated by the diagram at the right). The gray lines indicate the perceived category boundaries. The gray area is the hypothetical shape of the rhythmic category A. The dots identify the (interpolated) rhythms, crosses mark the mechanical ones (cf figure 2b).

\subsection{Representing rhythmic categories: The score space}

The syntactic aspects of rhythm can be formally described by considering rhythm to be the result of a metrical grammar (Longuet-Higgins 1978). Such a grammar describes a

(3) In this paper, all stimuli will be represented in the same format, eg the pattern A as $[0.25,0.50,0.25]$. 
rhythm as accommodated by a hierarchical tree of duple or triple subdivisions. The resulting metrical tree specifies an important recurrent time interval, the bar, and the way it is subdivided recursively. These subdivisions define several levels of regular isochronous pulse trains, so-called beats. The notion of tactus refers to the most perceptually salient level of the metre, the level at which musicians keep timing by counting.

However, we have to note that metre is just one structuring factor in rhythm (cf London 2001), and unfortunately a complete grammar (or formal theory) of rhythm does not exist. For our purposes, however, it is enough to consider the space of all possible music notations.

As the note durations in common music notation (CMN) can become very fine ( $1 / 32$ nd notes are in common use), and the ways to combine them are manifold (as was briefly discussed above), the space of all possible rhythmic sequences that can be notated in $\mathrm{CMN}$ is enormous, even when considering a brief rhythmic pattern. We will refer to this set of all possible discrete rhythmic notations as score space. ${ }^{(4)}$

\subsection{Categorisation as mapping from performance space to score space}

Categorisation can now be described as a mapping from a performance space into a score space. Such a transformation implies the partitioning of performance space into a set of equivalence classes: all points in performance space mapping to the same score belong to the same class. Thus, although rhythmic categories are named or labelled by a sequence of integers, they are characterised by a region, an area in performance space, as is depicted in figure 5 .

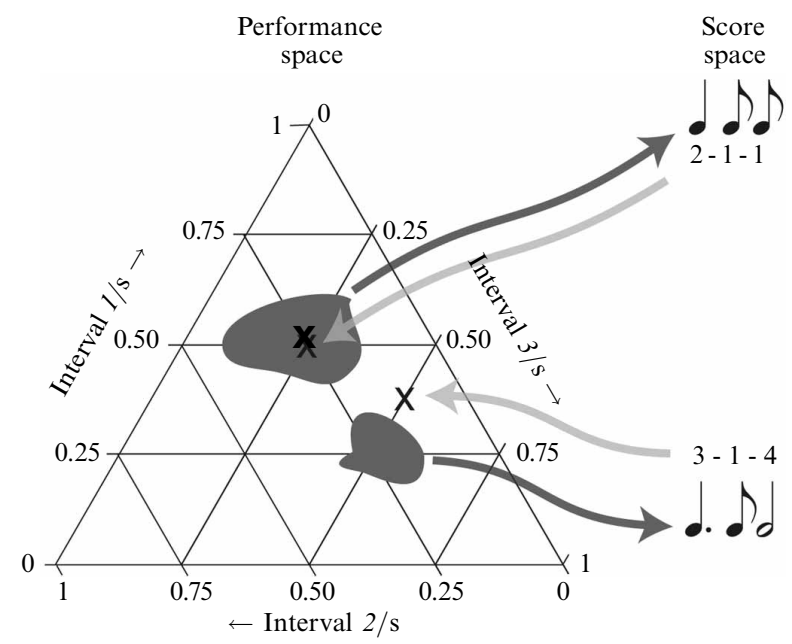

Figure 5. Hypothetical performance regions and their mapping to score space (dark gray arrows). Also, the mapping from score space to performance space (light gray arrows) with crosses indicating the mechanical rendition of the rhythmic category.

This brings us to the question how these regions or areas in performance space can be characterised. We will do this using concepts from abstract algebra and topology (see, eg, Fraleigh 1976 for an introduction). A first characterisation could be whether these areas are connected: no two separate regions in performance space form part of the same category (in score space). In that case, all renditions of a certain rhythmic category will be enclosed by the same area, supporting the notion that a single performance region represents a certain rhythmic category. More specifically, they may be convex: no interpolation between two performances of the same score will be perceived

(4) However, note that a score space is not a space in the strict mathematical sense (it has no metric). We use the term here loosely to refer to the domain of well-formed sentences in the grammar of CMN. 
as a different rhythm. In that case, the shape of the areas becomes necessarily bounded by straight lines: they are polygons that partition the performance space (cf Cemgil et al 2000).

Further issues that can be addressed in this way are the size of the areas and their shape. Are they larger for certain, possibly simpler rhythms? Are they symmetrical, or are the boundaries of expressive timing different for some intervals?

Finally, as a last example of the interesting issues that can be resolved when approaching categorisation as a mapping between spaces, we focus on quantisation. We use the term to refer to categorisation, as well as the transformation of the category back into a mechanical performance (ie both arrows in figure 5). The location of the mechanical performance need not necessarily lie inside the boundaries of its own region. In that case, it is no fixpoint: quantising an already quantised performance yields another category (in the examples given in figure 5, this holds for 2-1-1 but not for 3-1-4).

Before applying these concepts to our empirical data we will discuss our hypotheses and experimental setup.

\subsection{Hypotheses}

In this study, first we expect to find evidence for the formation of rhythmic categories, confirming in a systematic way the research described above. We hope to be able not only to observe the category boundaries, but also to investigate the size of the category-its size and shape representing the amount of expressive variation that is allowed while still being identified as belonging to that rhythmic category. Second, we expect to find an effect of metrical context [as was shown by Clarke (1987) for two rhythmic categories]. If categorical rhythm perception is indeed open to top-down cognitive influence, the categories might change in size and shape depending on the metrical context the rhythm is presented in.

Theories of metre perception predict an easier identification, coding, and recall for rhythmic patterns that are metrical-a notion that is, however, defined differently in the various models. Using the definition that metrical rhythms are those patterns that have onsets in important metrical positions (Palmer and Krumhansl 1990), contain subjective accents that align with a beat or clock (Povel and Essens 1985), minimise syncopation (Longuet-Higgins and Lee 1984), or couple well with hierarchically arranged oscillators (Large and Palmer 2002), one can hypothesise that temporal patterns that are in accordance with the metrical context in which they are presented (ie they induce the same metre) are more easily identified, priming the responses. We therefore expect increased consistency of responses when a metrical context is presented. Furthermore, a preference for a rhythmic interpretation of patterns in a duple metre (Drake 1993) would show up as a similarity between the duple and no-metre conditions.

Next to the available empirical data, well-performing models of quantisation (eg Longuet-Higgins 1976; Desain and Honing 1989) can be taken as theoretical, indirect indications for the shape of rhythmic categories. ${ }^{(5)}$ These models were shown to be quite accurate in describing the process of obtaining discrete rhythmic values from continuously varying note durations in a performed rhythm (Desain and Honing 1992). A visualisation of the behaviour of, for example, a connectionist quantiser that is based on a relaxation method (see Desain and Honing 1991, page 161) reveals that for a complete set of rhythms (a rhythm space as discussed above) there are larger areas around small integer ratios and smaller ones for more complex patterns, indicating more 'tolerance' or freedom in expressive interpretation for rhythms made up of small integers. Interpreting the watersheds between these so-called basins of attraction as the boundaries between categories, it predicts different shapes and sizes for the different

(5) Both quantisation models are able to derive the score shown in figure $1 \mathrm{~b}$ from the performed rhythm shown in figure la. 
rhythmic categories. ${ }^{(6)}$ Furthermore, this research suggests that categorisation is actually facilitated by the temporal context: a different metrical context can result in a different quantisation for the same performed temporal pattern (the example in figure 1 shows on a local scale with duple and triple divisions of the quarter note that two almost identical time intervals can give rise to two very different subjective durations). The Longuet-Higgins (1976) model arrives at a rhythmic interpretation by recursively subdividing a time interval in a duple or triple way until every onset in the pattern is close to the start or end of such a subdivision. This computational process can also be characterised by interpreting decision boundaries as the borders of the rhythmic categories. As such, both computational models address the same domain of cognitive functioning as we are trying to probe in our participants.

We will now describe, in more detail, the first identification experiment, designed to investigate this process of categorisation, and the second experiment, designed to pinpoint the influence of metrical context.

\section{Experiment 1}

\subsection{Methods}

3.1.1 Participants. The twenty-nine participants of experiment 1 were highly trained professional musicians and advanced conservatory students from Dutch conservatories and from the Kyoto City University of the Arts in Japan. They had received between 7 and 17 years of musical training and were paid for their participation.

3.1.2 Apparatus. The sounds were presented through headphones (Sennheiser HD 445) and generated by a Yamaha MU-90R synthesiser using General MIDI percussion sounds. The participants could adjust the loudness of the stimuli to a comfortable listening level. The synthesiser was driven by the POCO system (Honing 1990; Desain and Honing 1992) via the OMS MIDI driver, running on an Apple Macintosh G3. The same computer collected the responses via a CMN interface (see section 3.1.4).

3.1.3 Stimulus construction. The stimuli used in the experiment are a subset of all temporal patterns made up of four onsets adding up to a total duration of $1 \mathrm{~s}$. Since this performance space is still infinite, a subspace of patterns needs to be sampled. In this study, we use a temporal grid with a unit of $\frac{1}{19} \mathrm{~s}$ (about $0.053 \mathrm{~s}$ ). A sampling unit of $\frac{1}{19} \mathrm{~s}(0.053 \mathrm{~s})$ was used, ${ }^{(7)}$ with a minimum IOI of three units $\left(\frac{3}{19} \mathrm{~s}\right.$, ie $\left.0.158 \mathrm{~s}\right)$. Thus IOIs vary between $0.158 \mathrm{~s}$ and $0.684 \mathrm{~s}$ in steps of $0.053 \mathrm{~s}$. The choice of a prime unit prevents the induction of a fine metrical subdivison by the sampling itself. Furthermore, a minimum IOI was used to remain in the domain of musical note durations. In our experiments we choose a minimum duration of three time units, ie $\frac{3}{19} \mathrm{~s}(0.158 \mathrm{~s})$. The sampling needs to balance the size of the set with a fine enough resolution to still have a good representation of the continuous space of all possible performed rhythms. The 66 stimulus patterns used in the experiments are shown in figure 6.

Each pattern was embedded in a context consisting of a fixed sequence of eight $1 \mathrm{~s}$ time intervals used to induce the bar-level of a metre. We explicitly controlled for this, since rhythmic patterns are known to induce a beat or pulse (Povel and Essens 1985). The bar was marked by a 'low bongo' percussion sound (10 ms attack, $15 \mathrm{~ms}$ decay to $6 \mathrm{~dB}$ below peak level) on a Yamaha MU-90R synthesiser at MIDI velocity 64. ${ }^{(8)}$

(6) Relating these predictions to empirical data will be the topic for a future paper.

(7) Note that IOIs of stimulus patterns are presented not as multiples of $\frac{1}{19} \mathrm{~s}$ but are rounded to milliseconds for readability. This causes sometimes a small rounding error, eg a pattern summing to slightly less than $1 \mathrm{~s}$ duration. However, in the stimulus preparation and response analysis software a greater precision was used.

${ }^{(8)}$ In this paper, we represent performances (stimuli) always as a sequence of inter-onset intervals which makes the representation independent of the actual durations of the short percussive sounds. 


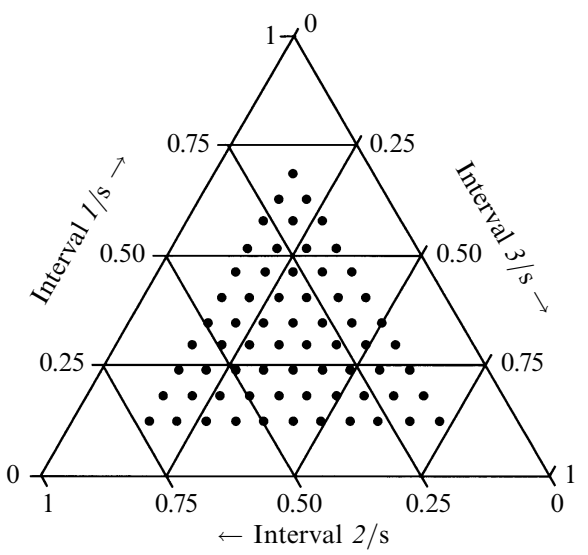

Figure 6. The sampling of the performance space as used in the experiments.

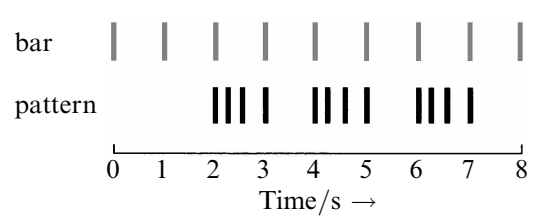

Figure 7. The stimulus pattern as used in experiment 1. Each line represents the onset of a percussive sound, the gray lines represent the metrical context against which the to be identified pattern (black lines) is repeated three times.

Within this metrical framework the stimulus patterns were embedded, being repeated three times (see figure 7). The onsets were marked by a 'high woodblock' percussion sound (1 ms attack to $5 \mathrm{~dB}$ above bar markers, $10 \mathrm{~ms}$ decay) at MIDI velocity 76 .

3.1.4 Response. For the identification task, a specially designed computer interface (see figure 8) was used, on which rhythms in CMN could be entered. Its design was guided by the responses obtained in a pilot study with an open response format on music paper. The computer interface allowed for a large set of time signatures, notes, and rests (ranging from a whole to a 1/32nd duration), dots, ties, and grouping in duplets, triplets, and quintuplets. Clicking buttons with the mouse allowed CMN to be formed. A correction button was provided to undo actions of button presses. An OK button signaled the participants confirmed response. An underlying grammar checked for appropriate music notations (ie rhythms of three intervals that add up to one bar, starting with a time signature), disabling buttons that would lead to a wrong notation. For example, after selecting a time signature at the start of the bar, all time signature buttons were disabled;

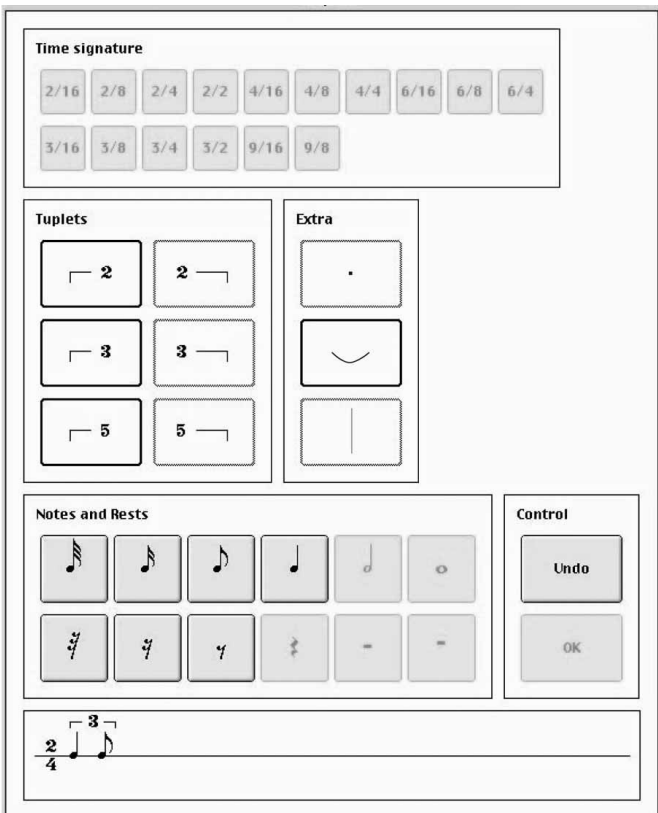

Figure 8. Music notation interface as used for the identification task. 
and after selecting a second dot after a note, the dot button was disabled until the next note was entered. ${ }^{(9)}$ The grammar disallowed only ill-formed responses, supporting millions of different rhythmic patterns, which were subsequently reduced to approximately ten thousand classes each represented by a unique integer sequence. The rhythmic responses were stored along with other data, like the number of times the correction button was used.

3.1.5 Procedure. The participants performed an identification task in which they were asked to notate the presented stimulus using the computer interface described above. The presentation of the stimuli and entering of the responses were self-paced, but each complete stimulus (ie three repetitions of the rhythm embedded in a context of eight bars - see figure 7) could only be listened to once. Each stimulus was presented once, in random order. Participants were instructed to think of the stimulus as if played by a percussionist, and to notate the score they thought was most likely used by the percussionist, this to prevent them from using extremely complex notation to write out the perceived expressive timing. This task was familiar to the participants as it is part of the standard solfège training at the music conservatory. The experiment took $45 \mathrm{~min}$ to complete; short breaks were allowed. To one single participant, the stimuli were presented multiple times, re-randomised in each of the six sessions, which took place with several days in between. Her responses were analysed separately.

3.1.6 Data preparation. All responses (ie music notations) were converted to an irreducible integer representation, with several music notation variants leading to the same integerratio representation. Response proportions (distribution of responses) were calculated for each stimulus. This constitutes the measurement data from which the various maps used in this paper are constructed, as is illustrated in figure 9. The bottom of this figure shows the performance space with the 66 stimuli used (cf figure 6). For three arbitrarily chosen stimuli the response proportions for the rhythms are shown (ie the three bars). The leftmost bar represents a stimulus that elicited only one response, the rightmost bar is an example where there is a clear maximal response, but alternatives were chosen as well by some participants. The middle bar is an example of the absence of a winning maximal response. The first map constructed from these data (see figure 9a) depicts maximal response proportions. This categorisation map, or time clumping map, shows 1-1-1 (red) for the leftmost bar, 1-1-2 (green) for the rightmost bar, and none (white) for the middle stimulus, since there is no response given significantly more often than any other for this performance. The second map (see figure $9 \mathrm{~b}$ ) shows the agreement in responses. This entropy map shows a relatively high value for the middle bar (red), as many different responses were given for that stimulus. For the other two stimuli there is much more agreement and the entropy is low (blue). ${ }^{(10)}$ The construction of the maps as continuous ternary contour plots was based on interpolated values in the stimulus space and visualised with JMP (version 3.2.2 by SAS). The details of the calculations used for the two maps are provided below.

\subsection{Analytical methods}

3.2.1 Measure of consistency. To determine the consistency within and between participants in the identification task we used a measure of uncertainty, which stems from information theory, and is used in physics as well as psychology (Garner 1975): the amount of noise in a signal can be expressed as the number of bits needed to encode

(9) As another example of the workings of this grammar, consider the partial result as entered by the user in figure 8: a triplet of a quarter note and an eighth note in $2 / 4$ metre. In this state, the button representing notes and rests that would exceed the duration of the bar are disabled (eg the quarter rest is disabled because still a third note needs to be added).

${ }^{(10)}$ The response proportions shown in the bottom of figure 9 are slightly idealised for instructive purposes. For detailed information on the actual responses (as depicted in the entropy and time clumping maps later in this paper), see www.nici.kun. nl/mmm/time.html. 
the information. We assume a multinomial distribution of responses. For a set of responses to the same stimulus, with $P_{i}$ denoting the response proportion for response $i$, and $n$ being the number of measurements (ie the number of participants times the number of repeated presentations), the Shannon entropy $E$ is defined as:

$$
E=-\sum_{i=1}^{n} P_{i} \log _{2} P_{i} .
$$

For example, if each of eight participants responds with a different notation to a single presentation of the same stimulus (or one participant responds differently on each of eight repeated trials), the entropy is three bits (much noise, low concordance). If the same response is given by all participants for that specific stimulus (or by one participant on all repeated trials), the entropy is 0 (high consistency).

We normalise this entropy measure by the maximally possible entropy, given $n$, the number of measurements, to obtain a measure which can be compared across conditions with a different number of measurements:

$$
E_{\mathrm{r}}=\frac{E}{\log _{2} n} .
$$

Since the relative entropy $E_{\mathrm{r}}$ is defined for each stimulus, the average relative entropy of a complete stimulus set is used when comparing the overall consistency of different groups of participants or of different conditions. Correlations between relative entropy distributions over the stimuli are used when comparing patterns of consistency of different groups of participants or of different conditions.

3.2.2 Confidence areas. For one stimulus, the response proportions for the various rhythmic patterns stem from a multinomial distribution. The rhythm that exhibits the largest response proportion was considered the winning category. However, one has to consider the statistical reliability of the statement that the probability of this category is indeed larger than any other, given the number of trials and the distribution, which is in general hard to do. However, because usually most proportions are close to zero and only two responses compete, the significance of their difference was tested as if they were independent. This approximation made it possible to indicate where in the stimulus space there was more than $90 \%$ confidence that one proportion was indeed the largest.

\subsection{Results}

The twenty-nine participants responded with 123 different categories. Of those responses, 21 rhythms were used only once by one participant. The pattern 1-1-1 was used most often as a response, in about $10 \%$ of all trials (in section 3.3.3, table 1 , a complete overview is given). In presenting the instruction, the nature of the identification task was difficult to make clear to some participants. Few professional musicians were lured into using extremely complex notation, not uncommon to the modern music repertoire. The time to get acquainted with the instruction and interface was minimal, and making use of the response interface proved easy. In the post-experiment interview, most participants stated that the task was tiring but not too hard to complete. None of the participants complained about the range of responses allowed by the user interface, not even when explicitly asked.

3.3.1 Is the task difficult? We first considered the difficulty of the task and the consistency of responses. To study this, one participant participated in this experiment six times. The relative entropy of the response distribution per stimulus for this participant is shown in figure 10a. Here, the blue areas indicate rhythms for which the same response was always given for the same stimulus (low entropy/high consistency), the red areas 
indicate patterns in which almost every presentation of the same stimulus elicited a different response (high entropy/low consistency). Whether the areas of high entropy represent unmusical performed rhythms or just highly ambiguous ones cannot be decided here, but both these high-entropy (high uncertainty) and low-entropy (high consistency) areas appear roughly similar when visualising the relative entropy of a large group of participants $(N=29)$ that received each stimulus once (see figure 10b). The correlation between the two entropy distributions is $0.47(p<0.001)$, indicating a response consistency within one participant which is analogous to the concordance between the participants.

A further validation of the interpretation of entropy as a measure of the difficulty of the identification task, as opposed to an interpretation as the size of intersubject differences in the perception of a performance, can be obtained by considering the number of corrections the participants used before arriving at the chosen response. The correction button was used on average 1.8 times per response. The correlation of the number of corrections over the stimuli with the entropy was $0.55(p<0.001)$, suggesting that difficulty of finding a proper response accounts indeed for a large part of the amount of entropy.

The areas of low entropy seem to focus around relatively simple rhythms like 1-1-1, 2-1-1, 1-2-1, and 1-1-2. For example, one of the most consistently identified stimuli is $[0.474,0.263,0.263]$ (in seconds) which was identified by twenty-eight of the twenty-nine participants as 2-1-1. The single participant also chose the same response six out of six times. The stimulus that prompted the widest range of responses was [0.211, 0.368, 0.421]. This pattern gave rise to 17 different rhythms, of which both 1-2-3 and 1-3-2 were most common $(14 \%)$, and many complex rhythms were chosen once. The single participant chose three different patterns in the six repeated trials of that same stimulus.

In conclusion we may say that the entropy patterns suggest that the task can be considered as not too difficult, especially in specific areas around relatively simple rhythms (ie rhythms with low integer ratios). Thus, meaningful analyses of the rhythmic categories identified come within reach.

3.3.2 Which rhythmic categories are identified where? To study this question, we examine the partitioning of the performance space according to the rhythms that are most frequently identified, considering all stimuli that attract the same response rhythm most often as belonging to the same category. Since we did not present participants with two preselected response categories as used in standard categorical perception investigations (Harnad 1987) but used semi-open response categories (cf Schulze 1989), we cannot use the conventional operational definition of the boundary of a category (ie examining the shapes of the identification and discrimination functions). ${ }^{(11)}$ Also, the commonly used confusion-matrix approach to the derivation of a discrimination measure is not applicable here, since correct response categories are not known. Figure 11 shows the maximally identified rhythms. This time clumping map reveals the apparent coagulation in rhythm perception. Colours represent rhythmic categories. Their music notation and integer representation are shown in the legend, which lists them in order of response proportion. Gray lines represent the boundaries between categories. Darker shades of colour indicate a larger proportion of participants who chose this identification (darkest shade marks 100\% agreement among participants). The white areas are an indication that there is less than $90 \%$ statistical confidence that one rhythmic category is identified most often.

As the reverse of perceptual categorisation, which assigns areas in performance space to the same score, these categories also form constraints on expressive musical performance.

(11) A check for an increased discrimination sensitivity around the category boundaries that are identified in our experiment will be left for future research. 
Each area signifies the freedom for the musician in choosing expressive timing, for were the performer to push the amount of expressive timing too much from the centre of the category, passing across a boundary, the audience would perceive another rhythm.

3.3.3 Analyses. This representation (see figure 11) allows us to investigate the topological aspects (like partitioning, shape, and position) of the responses and characteristics like symmetry and permutation.

We first consider the range of the categorisation transformation: the set of scores that are identified as the categorisation of a performance. Out of 123 different responses, there are 12 rhythms that are identified in the time clumping as achieving a maximal response. They cover $60 \%$ of the responses (see table 1). There are large differences in the sizes of these categories: simple patterns seem to account for a larger area of performance space. This is also reflected in the underlying response proportions. In table 1, the response proportions (over all 66 stimuli and all twenty-nine participants of experiment 1) of these patterns are listed, ordered by their response proportion. Only those patterns are listed that become the maximal response somewhere in the performance space.

Table 1. Response proportions in experiment 1.

\begin{tabular}{lll}
\hline Response & Proportion & Cumulative \\
\hline $1-1-1$ & 0.094 & 0.094 \\
$1-2-1$ & 0.080 & 0.174 \\
$2-1-1$ & 0.064 & 0.238 \\
$1-1-2$ & 0.060 & 0.298 \\
$2-3-1$ & 0.046 & 0.344 \\
$4-3-1$ & 0.043 & 0.387 \\
$3-1-2$ & 0.041 & 0.428 \\
$4-1-1$ & 0.039 & 0.467 \\
$1-3-2$ & 0.038 & 0.505 \\
$3-1-4$ & 0.031 & 0.536 \\
$1-1-4$ & 0.031 & 0.567 \\
$2-1-3$ & 0.028 & 0.595
\end{tabular}

Informally one can state that the response proportions reflect the simplicity of the pattern: patterns made up of low integer ratios (eg 1-1-1 and 1-2-1) take a large proportion of responses, and patterns like 5-4-7 or 1-5-3 are almost never given as response (in this case less than 0.001). However, since it is still all but clear which structural and perceptual factors contribute to perceived complexity of a rhythm (cf Tanguiane 1993; Shmulevich and Povel 2000), we will leave a discussion on the relation between our data and these theories to a separate occasion.

Second, note that the set of rhythmic sequences that appear in the time clumping map is not closed under permutation. Closed under permutation would mean that all orderings of 1-2-3 occur (ie 1-2-3, 2-3-1, 1-3-2, 3-1-2, 3-2-1, and 2-1-3). However, it turns out that certain patterns (eg 2-3-1 and 3-1-2) are present, but re-orderings of the intervals in the sequence (eg 1-2-3) are not. In that sense, rhythmic sequences in score space are not represented symmetrically in the responses. Only simple patterns, such as 1-1-2 appear in all possible orders. As an overall measure of this asymmetry of rhythmic sequences we consider the correlations between the 62 response proportions of patterns consisting of three different note durations (represented by $A, B$, and $C$ ) and their permutations. They are listed in table 2. Note that a reflection in time, ie $C-B-A$, though perceptually very different, is quite similar to the original rhythm $A-B-C$ in the amount it is being identified from performances. A reason for this could be that the tree structure needed 
Table 2. Correlations between response proportions of the pattern $A-B-C$ and its permutations.

Permutation Correlation with $A-B-C$

\begin{tabular}{ll}
\hline$B-A-C$ & 0.97 \\
$C-B-A$ & 0.94 \\
$B-C-A$ & 0.88 \\
$C-A-B$ & 0.76 \\
$A-C-B$ & 0.75
\end{tabular}

to metrically encode $A-B-C$ is the same for its reversal $C-B-A$, attracting a similar response proportion, while other permutations of $A-B-C$ might have a different metrical encoding, accounting for more differences between the amount of responses.

Third, we consider the partitioning of the performance space into areas. As it turns out, there is only one area for each rhythmic category. This means that in the representation adopted in this paper the rhythmic categories are connected. Thus, for any two performances perceived as instances of the same rhythm, there exists a path of arbitrarily fine interpolations connecting them such that all performances on this possibly curved path will be perceived as the same rhythm.

Fourth, the area for each category appears to be almost convex, which means that there is even a straight-line segment connecting two points within the same category that will lie completely inside that category. The map itself is therefore close to an idealised one with convex tiles in which the areas are replaced by convex polygons. We will refer to this situation as quasi-convex. To measure the closeness to convexity we compare each area to its convex hull (Cormen et al 1990), the smallest convex polygon circumscribing it. The boundaries of a convex area must necessarily be straight, as any bend would create a non-convex area on one side of the line.

Convexity of the categories is measured by the proportion of the surface area of the convex hull that is taken up by the category itself. It turns out that the categories cover on average $91 \%$ of the surface area of their convex hull, with a minimum of $71 \%$ and a maximum of $97 \%$. Thus, though one has to keep in mind that we average over participants, ${ }^{(12)}$ one could say that rhythmic categories are indeed convex. This has an important theoretical implication: with the categories being convex, a simple attractor model could, in principle, suffice to describe the behaviour observed; non-convexity would have ruled out these models. Furthermore, it implies that the boundaries between neighbouring categories, which are all convex, can only be formed by straight-line segments, ${ }^{(13)}$ important characteristic for modeling these data.

Fifth, we investigated whether mechanical performances of a rhythm are fixpoints of the quantisation transformation. If this is the case, quantisation can be applied twice without changing the outcome. In other words, any performance region will contain its rhythmic category mapped back to the performance space (see example 2-1-1 in figure 5). This turns out to be the case for all categories that attract a maximal response (ie all rhythms mentioned in table 1).

Sixth, we consider the position of the rhythmic category with regard to a prototype of the category: the mechanical performance. Judging this by the boundary,

(12) A set of convex individual response distributions might easily combine into a non-convex tiling for the whole population, for instance when a group of expert participants would distinguish a small rhythmic category which lies inside the larger, simpler category of less sophisticated users. It is thus all the more surprising that we find such highly convex tiling in our data, as the converse, non-convex distributions adding up to a convex one are much less likely.

(13) That is why the term tiling is appropriate. 

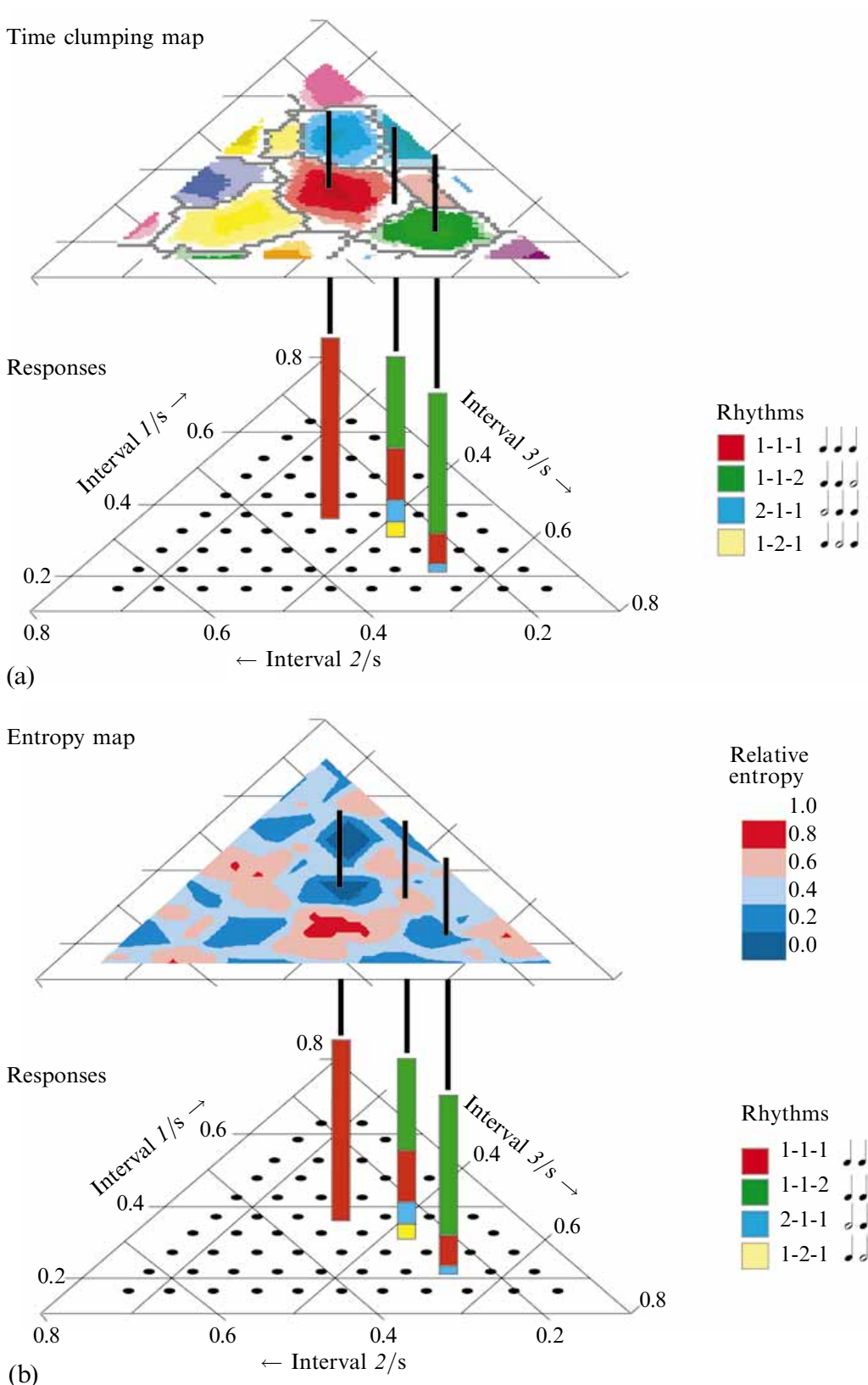

Rhythms

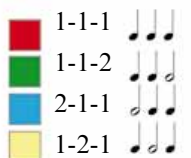

Figure 9. Construction of a time clumping map (a) and an entropy map (b) from the responses (see text for details).

in general the amount of allowed expressive timing deviation is distributed unevenly around the mechanical performance (marked with a black cross in figure 10) of the rhythmic category, usually with a slightly longer third interval. The same effect shows in the position of the areas marked with the darkest shade (ie the region where all participants identified a certain stimulus as the same rhythm). These regions also are not centred around the mechanical performance. This is most easily seen in the four patterns marked with a black cross in figure 10. For these rhythmic categories a nonmechanical stimulus attracted more responses than the mechanical one. This suggests that rhythmic categories may be labeled, but are not necessarily 

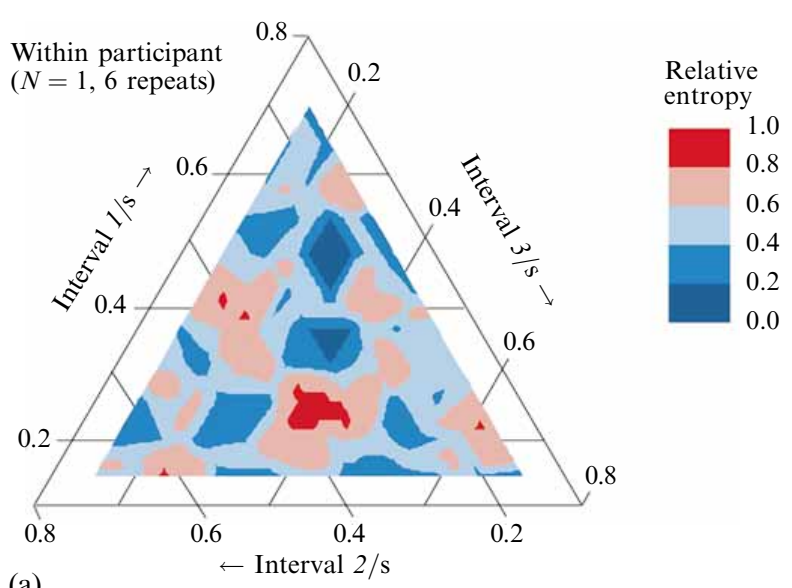

(a)
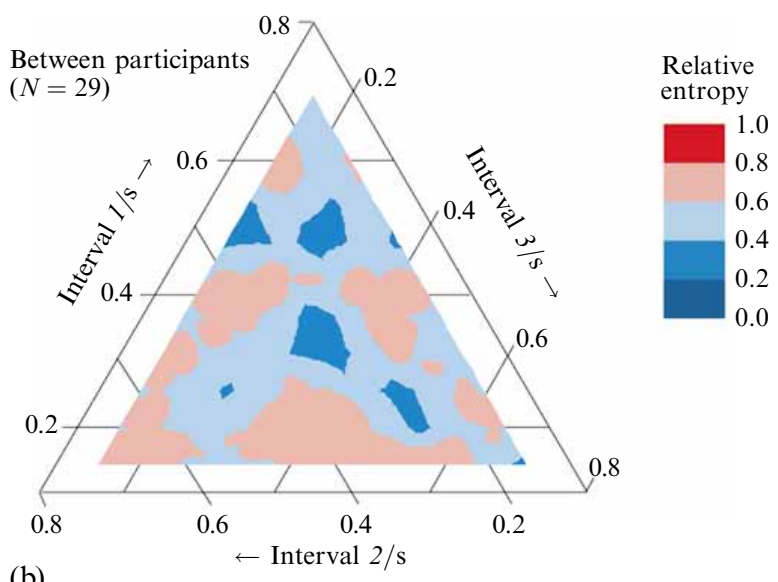

(b)

Figure 10. Entropy maps: entropy as a measure for consistency for the space of all possible three-interval temporal patterns (in seconds), (a) within participant $(N=1,6$ repetitions) and (b) between participants $(N=29)$. Blue indicates low-entropy/high-consistency areas, red represents high-entropy/high-uncertainty areas.

best prototyped, as a pattern of integers. ${ }^{(14)}$ They are actually shaped such as to reflect common expressive timing patterns in music performance. This also means that simple rounding methods can never model the process of rhythmic categorisation accurately.

Yet another way to estimate this effect is to judge the position of the centroids of the response distributions for the main rhythmic categories. ${ }^{(15)}$ Table 3 lists the performance centroids (in seconds) of the sensitive regions for the main rhythms, and the differences (in seconds) with a mechanical rendition. The distance from the mechanical performance reflects expected patterns of expressive timing for the various rhythms. Some regularities can be seen easily, for example a tendency to slow down at the end of a rhythm. Most of the IOIs are significantly different $(p<0.001)$ from their mechanical version when considered as marginal normal distributions, except the first interval

${ }^{(14)} \mathrm{Cf}$ the discussion in music performance research whether a mechanical performance can be used as norm in measuring expressive timing (Clarke 1995; Timmers and Honing 2002). Our findings suggest that a performance centroid (ie the most communicative rendition of a category) might be a better norm or reference.

${ }^{(15)}$ Because of boundary effects in the space of presented stimuli, they cannot be reliably estimated for the other categories. 

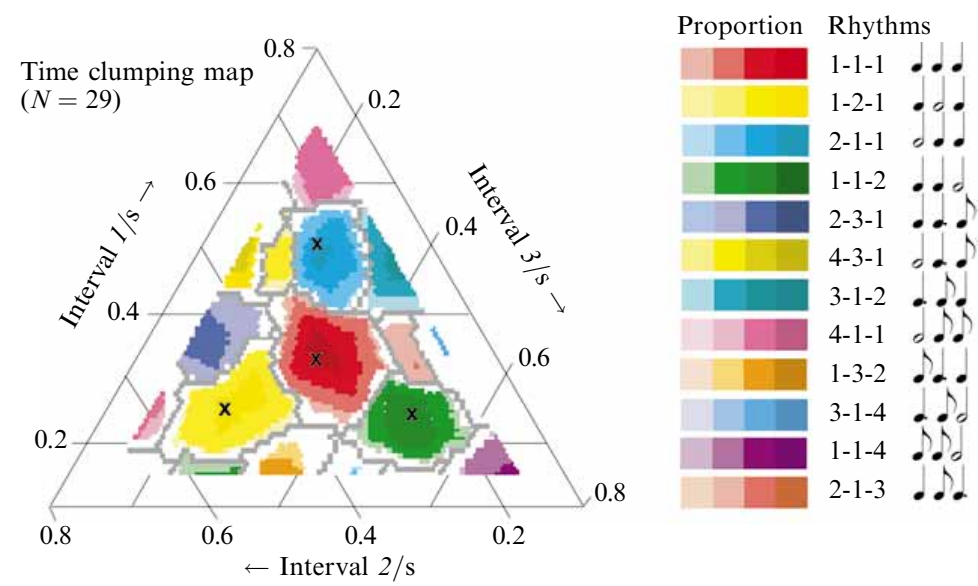

Figure 11. Time clumping map: transforming continuous time intervals (physical time) into rhythmic categories (perceived time). Colours represent the winning rhythmic categories (as identified in the 'Rhythms' legend at the right) with darker shades indicating a higher proportion of participants identifying it. Gray lines are category boundaries. In the white areas, there is less than $90 \%$ statistical confidence that there is one rhythmic category identified most often.

(a) Control (no metre)

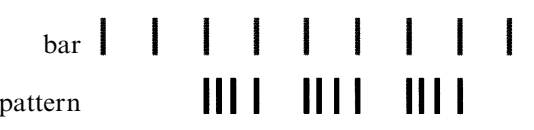

(b) Duple bar $||,||,||,||,, \mid$ metre pattern III I III I III I

(c) Triple metre

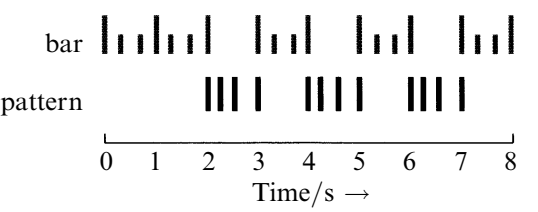

Figure 12. The stimulus pattern as used in the three conditions: (a) control (no metre), (b) duple metre, and (c) triple metre. Each line represents the onset of a percussive sound, the gray lines represent the metrical context against which the to be identified pattern (black lines) is repeated three times.

Table 3. Performance centroids and their difference (both in seconds) from a mechanical rendition.

\begin{tabular}{lll}
\hline Pattern & Performance centre & Distance from mechanical \\
\hline $1-1-1$ & {$[0.324,0.312,0.363]$} & {$[-0.009,-0.021,+0.030]$} \\
$1-2-1$ & {$[0.255,0.476,0.269]$} & {$[+0.005,-0.024,+0.019]$} \\
$2-1-1$ & {$[0.479,0.244,0.277]$} & {$[-0.021,-0.006,+0.027]$} \\
$1-1-2$ & {$[0.246,0.263,0.491]$} & {$[-0.004,+0.013,-0.009]$} \\
$2-3-1$ & {$[0.350,0.432,0.218]$} & {$[+0.017,-0.068,+0.051]$} \\
$4-3-1$ & {$[0.469,0.346,0.184]$} & {$[-0.031,-0.029,+0.059]$} \\
$3-1-2$ & {$[0.462,0.179,0.358]$} & {$[-0.038,+0.013,+0.025]$} \\
$4-1-1$ & {$[0.592,0.183,0.225]$} & {$[-0.075,+0.016,+0.058]$} \\
$1-3-2$ & {$[0.184,0.465,0.351]$} & {$[+0.018,-0.035,+0.018]$} \\
$3-1-4$ & {$[0.330,0.170,0.500]$} & {$[-0.045,+0.045,-0.001]$} \\
$1-1-4$ & {$[0.181,0.192,0.628]$} & {$[+0.014,+0.025,-0.039]$} \\
$2-1-3$ & {$[0.333,0.189,0.478]$} & {$[-0.000,+0.022,-0.022]$} \\
\hline
\end{tabular}



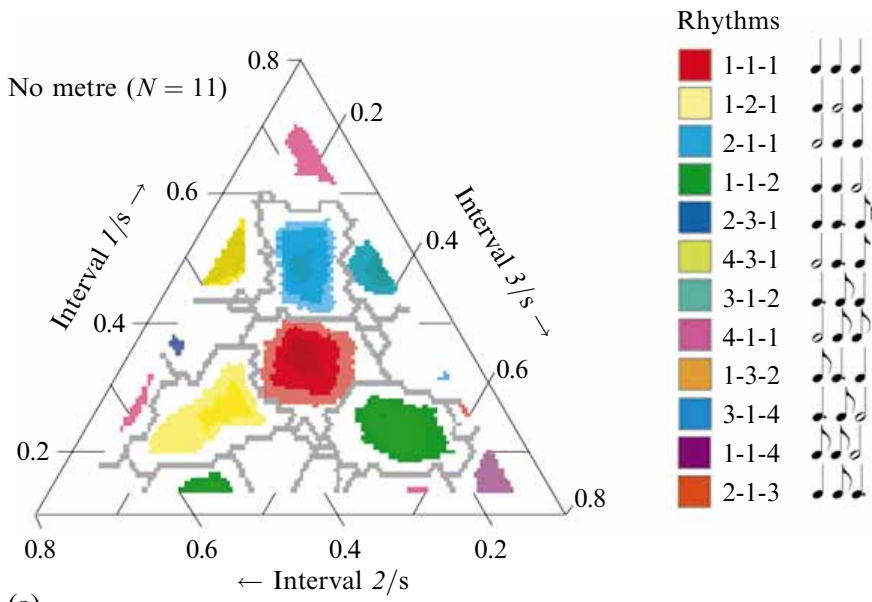

(a)
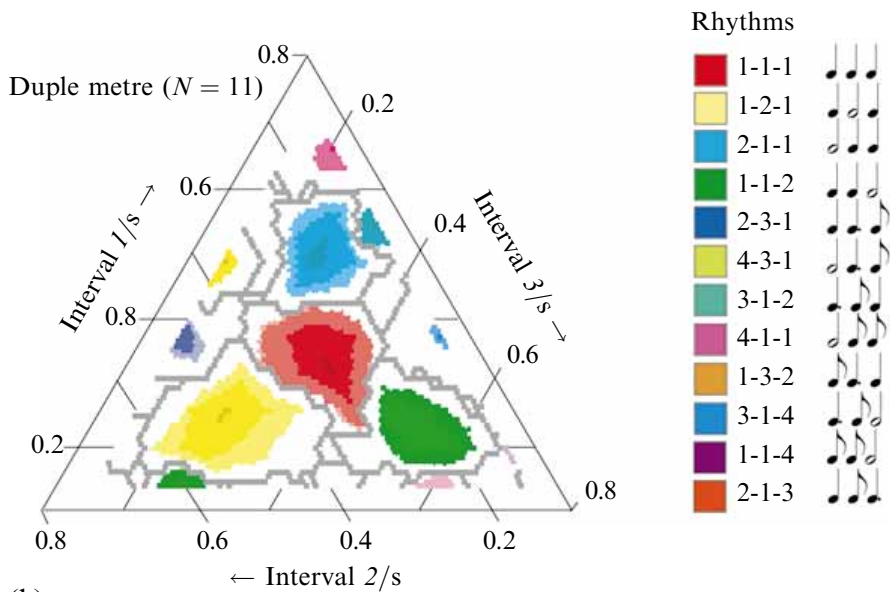

(b)
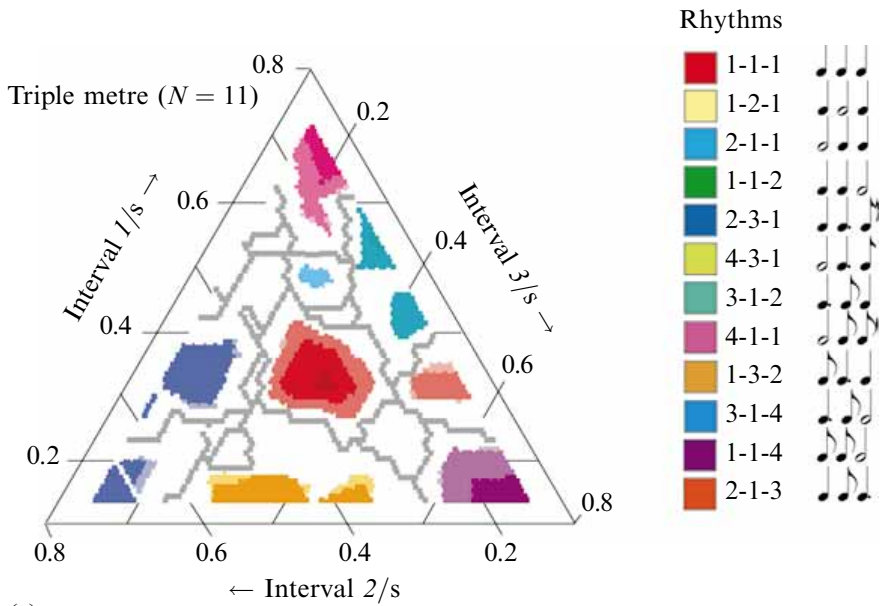

(c)

Figure 13. Time clumping maps: the effect of duple (b) and triple (c) metre as compared to the no-metre condition (a). Note the slight growth of the areas around simple rhythms like 1-1-2 and 1-2-1 in the duple-metre condition, and their complete disappearance in the triple-metre condition. (See www.nici.kun. $\mathrm{nl} / \mathrm{mmm} / \mathrm{time}$.html for an animation showing the changing categories.) 
of 2-1-3 and the last of 3-1-4, though they are all quite small relative to the sampling interval of $0.053 \mathrm{~s}$. The just noticeable difference (JND) of timing in an isochronous sequence of intervals of $0.333 \mathrm{~s}$ is about $0.006 \mathrm{~s}$ (Michon 1964; Friberg and Sundberg 1995 ) and can be compared with the $0.030 \mathrm{~s}$ timing deviation in the last note of the 1-1-1 pattern. Since a generative theory on the relation of rhythmic structure and expressive timing does not exist, we have no model to test against these data. However, the data support our argument that rhythm in score space is not well represented by its mechanical rendition in performance space, the performance centroid (interpreted as the most communicative rendition of a category) being a more likely candidate.

It is important to note that, because responses arise in competition, the centroids cannot be directly interpreted as the means of prior distributions of the timing of the rhythmic prototypes. Nevertheless, their position, in conformity to a well-known rule of music performance (slowing down at the end-cf Palmer 1997), is as expected.

The results presented above concern the categorisation of rhythmical patterns independently of context. As previous research (Clarke 1987) has shown that there might be an effect of metre on rhythmic identification, we conducted a second experiment in which we presented the same stimuli in different metrical contexts. This allows us to address the question whether the presence of a metre influences rhythmic identification.

\section{Experiment 2}

\subsection{Method}

4.1.1 Participants. Eleven participants took part in the experiment. All participants of experiment 2 had taken part in experiment 1 as well.

\subsubsection{Apparatus. Equipment was the same as in experiment 1.}

4.1.3 Stimulus construction. The stimuli used in the experiment were similar to the stimuli in experiment 1 . The empty bars were filled with a subdivision depending on the context condition. The two metrical context conditions (see figures $12 b$ and 12c) were created by filling the empty bars with a triple or duple subdivision. ${ }^{(16)}$ The beats were marked by a 'low bongo' percussion sound (10 ms attack to $8 \mathrm{~dB}$ below bar markers, $15 \mathrm{~ms}$ decay) at MIDI velocity 38. The control condition (see figure 12a) contained no subdivisions, exactly as in experiment 1.

4.1.4 Response. The same interface was used for collecting the responses as in experiment 1.

4.1.5 Procedure. The procedure and instruction were identical to those in experiment 1. The stimuli were presented in random order, blocked by context condition. The control condition was presented first, and the duple and triple conditions were presented in a random order.

\subsection{Analytical methods}

Data processing and analysis were similar to those in experiment 1. For comparing significance of differences between response proportions in multinomial distributions, we had to use an approximation: differences between probabilities of the same response in different conditions were tested individually, as if stemming from two binomial distributions.

\subsection{Results}

In total there were 158 different responses over all three conditions, but only 112 of those were used more than once. The duple-metre condition yielded 78 different responses, 90 different rhythmic patterns without metrical context, and 113 in triple context.

(16) In the light of the results of experiment 1, presenting a mechanical subdivision might not be the best way to prime a metre. However, a proper expressive rendition would be hard to construct without creating, eg, a dependence on musical style. Therefore we resorted to this solution. 
4.3.1 Is there an effect of metre? A metre can be defined as consisting of at least two levels of temporal structuring, for instance a bar subdivided in two or three equal beats (Martin 1972). We focus here only on the topmost two levels of metrical structure. This was studied by presenting the same rhythmic patterns preceded by a bar subdivided in two or three equal beats (duple and triple metre, respectively). As the participants were forced to choose a metre in which to notate their response, it is worthwhile inspecting these choices. In the condition without a metrical context, $84 \%$ of the responses were notated in a duple metre (like $4 / 4$ or $6 / 8$ ). There is no significant difference from the results of experiment 1 in which $81 \%$ of the responses were notated in a duple metre. The amount of duple-metre responses increased to $99 \%$ when duple metre was indeed presented, a highly significant increase $(p<0.001)$. In the case of a triple-metre context, only $5 \%$ of the responses were notated in duple metre, a highly significant decrease $(p<0.001)$, the rest used a triple metre (like $3 / 4$ or $9 / 8)$. These results suggest a successful manipulation of metrical context in the three conditions.

Let us first focus on the agreement between participants. The average relative entropy over all stimuli is 0.62 for the no-metre condition, 0.60 for the duple metre, and 0.68 for the triple metre. So, while slightly less consistent in the triple-metre condition, the overall consistency seems not to be affected by the absence or presence of a metrical context. This indicates that the difficulty of the task is, in general, not influenced by the availability of a metrical context. However, correlating the entropy distributions over the stimuli leads us to a different conclusion. Whilst the correlations between the entropy distributions are all significant $(p<0.05$ for the lowest correlation), they are still quite low ( 0.64 between no metre and duple metre, 0.28 between no metre and triple metre, and 0.23 between triple metre and duple metre). Given that the mean entropies are about the same for the three conditions, we conclude that the pattern of entropy distribution across the stimuli must be quite different in the three conditions. In other words, even though the conditions are of the same overall difficulty, the process of rhythmic identification is helped by presenting an appropriate metre and hindered by another one.

We may even assume that the appropriate metre would be the metre that participants construct mentally while listening to the stimulus in the no-metre condition. To test this hypothesis we defined for each stimulus the notions of appropriate metre as the metre that yields the highest agreement among subjects, or, in other words, the metrical context that made the transcription task less equivocal. If a stimulus is embedded in its most appropriate metre, the average entropy is 0.54 , as compared to 0.62 when no metre is provided. Similar figures $(0.60$ and 0.68$)$ arise when duple or triple metre is provided. So we can conclude that presenting the stimuli in a metrical context does not, by itself, ease the identification task. But, if the context is appropriate, it seems to help identification, as judged by the agreement among subjects.

Moving from the amount of agreement to chosen responses themselves, we can now present the effect of metric priming on the empirical response proportions.

The time clumping maps in figure 13 show the effect of metre on rhythmic identification in the three metrical contexts. Response proportions in the no-metre condition (figure 13a) correlate very highly $(0.94, p<0.001)$ with the responses in the duple-metre condition (figure 13b). This is as expected, since listeners tend to prefer a duple interpretation of rhythms when no metrical context is given (Longuet-Higgins 1976; Drake 1993). Notably, for the twelve-rhythms that are shown in the time clumping map, only for three rhythms there is a significant difference in response proportions between the duple-metre and the no-metre conditions. Table 4 lists the response proportions over all (66) stimuli and all (eleven) participants in the no-metre condition, plus the difference between the conditions, listing only those patterns that become the maximal response somewhere in the performance space. The table is ordered according 
Table 4. Response proportions in the no-metre condition and the differences between conditions.

\begin{tabular}{lcccc}
\hline Response & No metre & $\begin{array}{c}\text { Duple metre } \\
\text { versus } \\
\text { no metre }\end{array}$ & $\begin{array}{c}\text { Triple metre } \\
\text { versus } \\
\text { no metre }\end{array}$ & $\begin{array}{c}\text { Triple metre } \\
\text { versus } \\
\text { duple metre }\end{array}$ \\
\hline $1-3-2$ & 0.035 & $-0.030 * * *$ & $0.036 * *$ & $0.066 * * *$ \\
$1-1-4$ & 0.029 & -0.011 & $0.029 * *$ & $0.040 * * *$ \\
$2-3-1$ & 0.037 & -0.009 & $0.026 *$ & $0.035 * * *$ \\
$4-1-1$ & 0.036 & -0.006 & $0.029 * *$ & $0.035 * * *$ \\
$3-1-2$ & 0.036 & -0.004 & $0.019 *$ & $0.023 *$ \\
$2-1-3$ & 0.024 & 0.001 & 0.014 & 0.013 \\
$1-1-1$ & 0.084 & 0.004 & -0.012 & -0.016 \\
$4-3-1$ & 0.040 & -0.004 & $-0.029 * * *$ & $-0.025 * * *$ \\
$3-1-4$ & 0.026 & $0.016 *$ & $-0.018 * *$ & $-0.034 * * *$ \\
$1-1-2$ & 0.063 & 0.008 & $-0.039 * * *$ & $-0.047 * * *$ \\
$2-1-1$ & 0.066 & 0.008 & $-0.048 * * *$ & $-0.056 * * *$ \\
$1-2-1$ & 0.074 & $0.039 * *$ & & $-0.096 * * *$ \\
$* p<0.05 ; * * p<0.01 ; * * * p<0.001$. & & & \\
\hline
\end{tabular}

to the size of the differential effect of triple metre versus duple metre (rightmost column), the topmost response gaining most when presented in a triple-metre context (approximate significance of the shifts in response proportions is indicated by asterisks). This suggests a similarity of the duple metre, when primed, to the duple metre as preferentially induced in the mind of the listener when absent in the stimulus.

However, behaviour in the triple-metre condition (figure 13c) differs significantly more $(p<0.01)$ than the duple-metre from the no-metre conditions as the correlation is 0.70 (as compared to 0.94 between duple metre and no metre). And, as expected, it is even more different from the duple-metre condition (correlation 0.53). This is reflected in the significance of the differences between response proportions (as shown in table 4): only for two rhythms these shifts are not significant. Pattern 1-1-1, we can assume, is such a simple and well-known subdivision that even adding a duple metre, in which case it appears syncopated, does not make it less readily identifiable. The other pattern that is indifferent to metrical context, 2-1-3, is an interesting one. It is ambiguous in the sense that it fits both metres well. Thus, adding either duple metre or triple metre does not make it harder to identify, it just achieves another metrical interpretation. This is reflected in the relative position of the 2-1-3 area in the performance space: in duple metre it is centred around [0.341, 0.179, 0.480], exhibiting a long first note, while a triple-metre context yields a longer second note. In this case [0.329, $0.196,0.476]$ is the performance centroid, apparently the most communicative rendition of the rhythm 2-1-3 in a triple-metre context.

Thus, while an ambiguous rhythm may receive the same response proportions irrespective of metre, the expected expressive timing may differ. This is an important point for any theory of rhythm perception, as the interaction between the processes of rhythmic categorisation and beat induction need to be made clear. Apart from the two patterns 1-1-1 and 2-1-3, most rhythms are very sensitive to metric priming as exhibited by their response proportions. The largest change occurs for 1-2-1, which, albeit syncopated in duple metre, is readily identifiable in that metre. In the triplemetre case this pattern becomes almost impossible to recognise. The largest change in the opposite direction is exhibited by the 1-3-2 rhythm. This pattern becomes easier to recognise when primed by triple metre; primed by duple metre, it almost disappears. The results presented show the large overall effect of metric priming considering the response proportions over all of the stimulus space. 
Furthermore, the effect of metre also completely changes the tiling of the performance space (see figure 13). Some simple rhythmic categories that were identified quite often for a specific metre-less stimulus, and that were even more salient in the context of a duple metre, disappear completely in triple metre. To give an example: the stimulus that is perceived most differently in the two metres is [0.210, 0.474, 0.316]. In duple metre it is heard mostly as $1-2-1$ (64\% of the responses; with another $10 \%$ identifying it as 1-3-2), in triple metre it is interpreted mostly as $1-3-2$ (36\% of the responses, not one participant identifying it as 1-2-1).

So, in summary we conclude that there is a strong effect of metre on rhythmic identification.

\section{Discussion}

Psychological research focusing on cognitive processes often restricts itself to the study of the effects of a few conditions, when it is faced with the sheer enormity of the set of possible stimuli that may be used. In this paper, we have tried to directly address this difficulty in more encompassing way. By carefully formalising restricted spaces of stimuli and responses and systematically probing them, it turned out to be possible to contain the combinatorial explosion of possibilities and yet arrive at general statements on the cognitive process itself, instead of being constrained to ad-hoc statements about the differences between a few stimuli. Concerning the categorisation of temporal patterns, we have concluded from our study that the space of performances is partitioned into a small set of connected rhythmic regions. As a consequence, a model that chooses the smallest distance between performance and a set of rhythmic categories (nearest neighbour) might be appropriate. This set is not closed under sequential permutation. The categories differ in size, according to the complexity of the rhythm, and their location is not centred around the position of a mechanical rendition of the category. The shape of a rhythmic category in performance space is quasi-convex. This tells us something about the fabric of the underlying rhythm space and the processes that access it, as convex compartments occur quite often in nature, like the surfaces between soap bubbles. These mechanisms are commonly formalised by non-symbolic methods like differential equations, complex dynamics, relaxation networks, and attractors. Thus such formalisms may be the best candidates for a successful modeling of rhythmic categorisation.

The fact that one is now able to formalise the rhythmic categories facilitates research on this abstract level towards a computational model that captures behaviour so fundamental to rhythmic perception. Both its importance, and the complexity of the modeling task are demonstrated by two facts. First, without rhythmic categorisation there would be no reference against which to judge the expressive duration of a note: one would not be able to appreciate the difference between a deadpan and an expressive performance. And, second, too much and too strict categorisation would cause a loss of timing information and the difference between a deadpan and an expressive performance would not even be noticeable.

Turning to the effect of metric priming, Clarke (1987) had already demonstrated an effect of metre on the position of the category boundary for a few rhythmic patterns. In this investigation, we have shown the effect for a systematic set of temporal patterns and their clumping into rhythmic categories. The results for the different contexts had to be subsequently interpreted as a large and robust effect of metric priming. Thus rhythmic categorisation depends on a pre-established cognitive framework of time structuring, a main finding of this investigation. In the absence of a metrical context a duple metre was clearly preferred, as the responses were similar to those with a duple-metre prime. The effect of priming can be partly explained on the basis of the temporal structure of the responses: the location of note onsets on a 
temporal grid. This is in accordance with theories that stress the importance of the role of metre in the mental representation of rhythm (Longuet-Higgins 1976; Povel and Essens 1985), sometimes even so far as to state that rhythm only exists under a metrical interpretation.

The question of the relation between the rhythmic-categorisation process and the metre-induction processes is still open. Are these processes modular and conducted in sequential order, or are they better understood as one integrated process? Most metreinduction studies (Longuet-Higgins and Lee 1984; Povel and Essens 1985; Parncutt 1994) ${ }^{(17)}$ have used readily categorised rhythmic time intervals as input, with the exception of Longuet-Higgins (1976) who presents an algorithm that induces metre while categorising the rhythm. Our finding that the rhythmic categories depend on metre can only demonstrate that the categorisation process is open to induce metre. However, since the areas of timing that define a category for the ambiguous rhythms, which fit both metres equally well, were shown to be systematically different, the timing information itself might provide a cue for metre induction. This communication of metre by expressive timing (cf Sloboda 1983) suggests that metre induction and rhythmic categorisation are closely interrelated processes. Furthermore, we found that, while presenting one or the other metre did not increase participant agreement as compared to presenting no metre, presenting an appropriate metre for each response did increase the conformance of the responses. Thus, presence of a metrical context eases the formation process of rhythmic categories, which again is in agreement with a model such as the one formulated by Longuet-Higgins $(1976,1979)$ which, while categorising, grafts a representation of musical rhythm onto the framework of a preestablished metre.

As metre is not the only contextual aspect that may influence rhythm identification, we suggest that other aspects of rhythm, such as global tempo (Handel 1993), loudness accents, articulation, and melodic structure (Tekman 1997), remain worthy of investigation (Honing 2002). The methodology that was developed and the results that were presented can contribute to a fuller understanding and validation of the computational models of quantisation and the theoretical accounts of rhythm perception (LonguetHiggins 1976; Desain and Honing 1991; Large and Kolen 1995) which are still in urgent need of empirical support (Desain et al 1998). And, in the larger scheme, it appears that the topic of categorisation has a much wider relevance, as it reflects the transition from sub-symbolic to symbolic mental representations and thus forms a bridge from perceptual processes to cognitive ones, with rhythm perception being an intriguing domain of where these levels of representation meet. ${ }^{(18)}$

Acknowledgments. This research was funded by the Netherlands Organization for Scientific Research (NWO). Support was also provided by the Canon Foundation. Experiments were conducted by Rinus Aarts, Chris Jansen, and Maki Sadakata, at the Nijmegen Institute for Cognition and Information (NICI), and at the Kyoto City University of the Arts. We are grateful to John A Michon, Mari Riess Jones, and two anonymous reviewers for their thoughtful comments on an earlier draft, greatly improving the current presentation. Finally, we like to thank Huub van Thienen for implementing the CMN user-interface, Kathleen Jenks for correcting our English, and Torsten Anders, Maki Sadakata, and Paul Trilsbeek for constructing the web demonstration available at www.nici.kun. nl/mmm/time.html.

(17) See Desain and Honing (2001) for a study of the first two models referred to, and their success in explaining the empirical data presented in this paper.

${ }^{(18)}$ For an appendix containing all experimental data, audio examples, and animations, see www.nici.kun.nl/mmm/time.html 


\section{References}

Cemgil T, Desain P, Kappen B, 2000 "Rhythm quantization for transcription" Computer Music Journal 24(2) $60-76$

Clarke E F, 1987 "Categorical rhythm perception: an ecological perspective", in Action and Perception in Rhythm and Music Ed. A Gabrielsson (Stockholm: Royal Swedish Academy of Music) pp 19-33

Clarke E F, 1995 "Expression in performance: generativity, perception and semiosis", in The Practice of Performance: Studies in Musical Interpretation Ed. J Rink (Cambridge: Cambridge University Press) pp $21-54$

Clarke E F, 1999 "Rhythm and timing in music", in Psychology of Music Ed. D Deutsch, 2nd edition (New York: Academic Press) pp 473-500

Clarke E F, 2000 "Categorical rhythm perception and event perception", in Proceedings of the International Music Perception and Cognition Conference Department of Psychology, Keele University, CD-Rom

Cormen T H, Leiserson C E, Rivest R L, 1990 Introduction to Algorithms (Cambridge, MA: MIT Press)

Desain P, Honing H, 1989 "Quantization of musical time: a connectionist approach" Computer Music Journal 13(3) $56-66$

Desain P, Honing H, 1991 "Quantization of musical time: a connectionist approach", in Music and Connectionism Eds P M Todd, D G Loy (Cambridge, MA: MIT Press) pp 150-167

Desain P, Honing H, 1992 Music, Mind and Machine: Studies in Computer Music, Music Cognition and Artificial Intelligence (Amsterdam: Thesis)

Desain P, Honing H, 2001 "Modeling the effect of meter in rhythmic categorisation: Preliminary results" Journal of Music Perception and Cognition 7(2)

Desain P, Honing H, Van Thienen H, Windsor L W, 1998 "Computational modeling of music cognition: Problem or solution" Music Perception 16(1) 151 - 166

Desain P, Windsor L (Eds), 2000 Rhythm Perception and Production (Lisse: Swets and Zeitlinger)

Drake C, 1993 "Reproduction of musical rhythms by children, adult musicians and adult nonmusicians" Perception \& Psychophysics 53 25-33

Fraisse P, 1982 "Rhythm and tempo", in Psychology of Music Ed. D Deutsch (New York: Academic Press) pp $149-180$

Fraleigh J B, 1976 A First Course in Abstract Algebra (Reading, MA: Addison-Wesley)

Friberg A, Sundberg J, 1995 "Time discrimination in a monotonic, isochronous sequence" Journal of the Acoustical Society of America $982524-2531$

Gabrielsson A, 1999 "The performance of music", in Psychology of Music Ed. D Deutsch, 2nd edition (New York: Academic Press) pp 501-602

Garner W R, 1975 Uncertainty and Structure as Psychological Concepts (New York: John Wiley and Sons)

Handel S, 1993 "The effect of tempo and tone duration on rhythm discrimination" Perception \& Psychophysics $\mathbf{5 4} 370-382$

Harnad S, 1987 Categorical Perception. The Groundwork of Cognition (Cambridge: Cambridge University Press)

Honing H, 1990 "POCO: an environment for analysing, modifying, and generating expression in music", in Proceedings of the International Computer Music Conference (San Francisco, CA: Computer Music Association) pp 364-368

Honing H, 2002 "Structure and interpretation of rhythm and timing" Tijdschrift voor Muziektheorie $7227-232$

Jones M R, 1990 "Musical events and models of musical time", in Cognitive Models of Psychological Time Ed. R A Block (Hillsdale, NJ: Lawrence Erlbaum Associates) pp 207-240

Large E W, Jones M R, 1999 "The dynamics of attending: How people track time-varying events" Psychological Review 10(1) $119-159$

Large E W, Kolen J F, 1995 "Resonance and the perception of musical meter" Connection Science $6177-208$

Large E W, Palmer C, 2002 "Perceving temporal regularity in music" Cognitive Science 26 1-37

Le Grand Y, 1968 Light, Color and Vision 2nd edition (London: Chapman and Hall)

Lerdahl F, Jackendoff R, 1983 A Generative Theory of Tonal Music (Cambridge, MA: MIT Press)

Livingstone K R, Andrews J K, Harnad S, 1998 "Categorical perception effects induced by category learning" Journal of Experimental Psychology: Learning, Memory, and Cognition 24 $732-753$

London J, 2001 "Entry on 'Rhythm'", in The New Grove Dictionary of Music and Musicians 2nd edition (London: Macmillan) 
Longuet-Higgins H C, 1976 "The perception of melodies" Nature 263 646-653 (reprinted in Longuet-Higgins 1987)

Longuet-Higgins H C, 1978 "The grammar of music" Interdisciplinary Science Reviews 3 148-156 (reprinted in Longuet-Higgins 1987)

Longuet-Higgins H C, 1979 "The perception of music" Proceedings of the Royal Society of London, Series B $205307-322$ (reprinted in Longuet-Higgins 1987)

Longuet-Higgins H C, 1987 Mental Processes (Cambridge, MA: MIT Press)

Longuet-Higgins H C, Lee C S, 1984 "The rhythmic interpretation of monophonic music" Music Perception $1424-441$ (reprinted in Longuet-Higgins 1987)

Martin J G, 1972 "Rhythmic (hierarchic) versus serial structure in speech and other behaviour" Psychological Review $79487-509$

Michon J A, 1964 "Studies on subjective duration 1. Differential sensitivity on the perception of repeated temporal intervals" Acta Psychologica $22441-450$

Michon J A, Jackson J L, 1985 Time, Mind, and Behavior (Berlin: Springer)

Nakajima Y, 1987 "A model of empty duration perception" Perception 16 485-520

Palmer C, 1997 "Music performance" Annual Review of Psychology 48 115-138

Palmer C, Krumhansl C L, 1990 "Mental representations of musical meter" Journal of Experimental Psychology: Human Perception and Performance 16 728-741

Parncutt R, 1994 "A perceptual model of pulse salience and metrical accent in musical rhythms" Music Perception $11409-464$

Povel D J, Essens P, 1985 "Perception of temporal patterns" Music Perception 2411 - 440

Repp B H, 1984 "Categorical perception: Issues, methods, findings", in Speech and Language, Advances in Basic Research and Practice 10 Ed. N J Lass (New York: Academic Press) pp 242-335

Saunders B A C, Brakel J van, 1997 "Are there nontrivial constraints on colour categorization" Behavioral and Brain Sciences $20167-228$

Schulze H H, 1989 "Categorical perception of rhythmic patterns" Psychological Research 51 10-15

Shmulevich I, Povel D, 2000 "Complexity measures of musical rhythms", in Rhythm Perception and Production Eds P Desain, W L Windsor (Lisse: Swets \& Zeitlinger) pp 239-244

Sloboda J A, 1983 "The communication of musical meter in piano performance" Quarterly Journal of Experimental Psychology $35377-396$

Sternberg S, Knoll R L, Zukofsky P, 1982 “Timing by skilled musicians", in Psychology of Music Ed. D Deutsch (New York: Academic Press) pp $181-239$

Tanguiane A S, 1993 Artificial Perception and Music Recognition (Berlin: Springer)

Tekman H G, 1997 "Interactions of perceived intensity, duration, and pitch in pure tone sequences" Music Perception $14281-294$

Timmers R, Honing H, 2002 "On music performance, theories, measurement and diversity" Cognitive Processing (International Quarterly of Cognitive Sciences) 1/2 1-19

Todd N P M, 1992 "The dynamics of dynamics, a model of musical expression" Journal of the Acoustical Society of America $\mathbf{9 1} 3540-3550$

Vos P, Handel S, 1987 "Playing triplets: facts and preferences", in Action and Perception in Rhythm and Music Ed. A Gabrielsson (Stockholm: Royal Swedish Academy of Music) pp 35-47 


\section{PERCEPTION}

VOLUME 322003

www.perceptionweb.com

Conditions of use. This article may be downloaded from the Perception website for personal research by members of subscribing organisations. Authors are entitled to distribute their own article (in printed form or by e-mail) to up to 50 people. This PDF may not be placed on any website (or other online distribution system) without permission of the publisher. 